\title{
Low-complexity pixel detection for images with misalignment and interpixel interference in holographic data storage
}

\author{
Chi-Yun Chen, Chih-Cheng Fu, and Tzi-Dar Chiueh* \\ Graduate Institute of Electronics Engineering and Department of Electrical Engineering, \\ National Taiwan University, Taipei 10617, Taiwan \\ ${ }^{*}$ Corresponding author: chiueh@cc.ee.ntu.edu.tw
}

Received 30 June 2008; revised 1 November 2008; accepted 6 November 2008; posted 11 November 2008 (Doc. ID 98084); published 12 December 2008

\begin{abstract}
This paper presents an efficient solution to recovering data pixels of images that have undergone optical and electrical channel impairments in holographic data storage systems. The channel impairments considered include interpixel interference, three types of misalignment, and noise. The proposed misalignment-compensation scheme, consisting of realignment and rate conversion, can effectively eliminate misalignment with more than $84 \%$ reduction in additions and $74 \%$ reduction in multiplications. In addition, several low-complexity techniques are introduced to reduce the complexity of a two-dimensional maximum a posteriori pixel detection method by up to $95 \%$ and do so with negligible degradation in detection performance. (C) 2008 Optical Society of America

OCIS codes: $\quad 100.3010,100.3020,200.3050,200.4960,210.2860,100.0100$.
\end{abstract}

\section{Introduction}

Page-oriented memories (POMs), which are considered as the next-generation optical storage, have garnered a great deal of interest from researchers worldwide. Recent researches in holographic data storage (HDS), the most up-and-coming candidate for POMs, are driven by the promise of extremely high capacity and ultrafast data transfer rate [1-4]. HDS systems record information in a twodimensional (2D) format rather than in onedimensional (1D) bit streams along a spiral, as in CD/DVD systems. Consequently, HDS throughput is much higher as data is accessed in pages. Additionally, by storing data throughout the volume of the storage medium, HDS systems have elevated optical storage capacity to the terabyte level.

Unfortunately, boosted storage density evokes severe crosstalk among data pixels and physical limits on mechanical/electrical/optical components introduce misalignment in retrieved images. In general,

0003-6935/08/366784-12\$15.00/0

(C) 2008 Optical Society of America signals in the HDS channel suffer from three kinds of impairments, i.e., pixel misalignment, interpixel interference (IPI), and noise. Any small error in optical fabrication, mechanical vibration, and media deformation can lead to severe misalignment effects, namely magnification, translation, and rotation, between the recorded images and the retrieved images. IPI can result from any or any combination of the following: band-limiting optical apertures, diffraction, defocus, and other optical aberrations. In addition, optical and electrical noise increases bit-error-rate (BER) and reduces the effective storage capacity. Therefore, an effective pixel detection scheme is essential to the success of the HDS systems.

Misalignment in retrieved images can substantially corrupt signal fidelity and thus must be dealt with during signal detection. An iterative detection method with decision feedback and consideration of the translation effect was proposed in [5] . The feedback process is repeated for each possible translation angle, making it quite time-consuming. On the other hand, a similar signal detection method considering the rotation effect was proposed in [6]. Note that both 
designs can handle only one type of misalignment. To compensate for all three types of misalignment, the authors in [7] proposed an oversampling approach capable of recovering arbitrarily misaligned data pages. This approach makes use of the equalization concept to eliminate both misalignment and IPI. However, the linear equalizer in this method can not effectively eliminate the nonlinear distortion inherent in HDS channel.

Interpixel interference (IPI) is no doubt a major detriment to signal detection in POMs. There have been a number of approaches proposed to deal with IPI, specifically for the HDS systems. Linear minimum mean squared error (LMMSE) equalization [8] is one popular solution, but it cannot efficiently handle the nonlinear distortion inherent in the HDS channel. Several detection algorithms are a simplification of the maximum likelihood page detection (MLPD) method and thus provide only lessthan-optimal BER performance [9-11]. Among these techniques, the two-dimensional maximum $a$ posteriori (2D-MAP) detection [9] achieves the best BER performance, which is very close to the MLPD bound. However, high computational complexity of the 2DMAP renders its direct implementation impractical.

In this paper, we propose a low-complexity pixel detection solution for the HDS systems with the following features:

- a low-complexity compensation filter capable of realigning three misalignment effects and converting sample rate, and

- four complexity-reduction techniques that eliminate $95 \%$ of the computational complexity in the original 2D-MAP algorithm.

The rest of this paper is organized as follows. Section 2 describes the HDS system and the channel model. In Section 3 we propose a misalignmentcompensation scheme including realignment and rate conversion to compensate for all three kinds of misalignment and adjust the sample rate. After misalignment compensation and pixel matching, the signals are detected by a modified 2D-MAP scheme with four proposed complexity-reduction techniques, which are introduced in Section 4. The simulation results shown in Section 5 clearly indicate that our proposed solution indeed achieves near-optimal performance with much reduced complexity. Finally, Section $\underline{6}$ concludes this paper.

\section{System Overview}

\section{A. Channel Model}

The channel model adopted in this work is based on the traditional 4- $f_{L}$ system [11], whose recording and retrieving processes are illustrated in Figs. 1(a) and 1(b), respectively. In recording, an object beam passes through the spatial light modulator (SLM), containing a $2 \mathrm{D}$ binary information image, and the Fourier lens. Then, a corresponding refer-

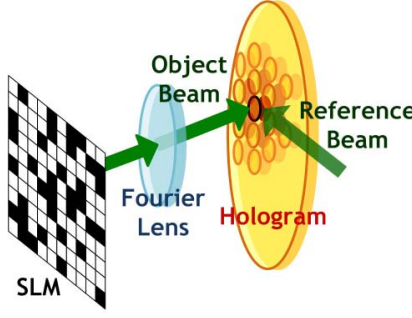

(a)

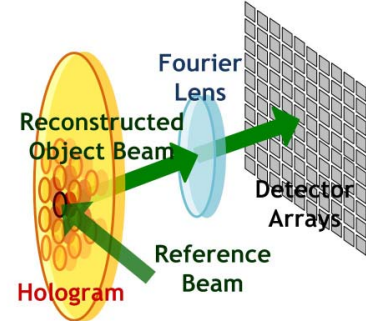

(b)
Fig. 1. (Color online) Holographic data storage system: (a) recording process and (b) retrieving process.

ence beam interferes with the object beam to form an interference pattern, namely a hologram, in the storage medium. By altering one or more characteristics of the reference beam, e.g., angle, wavelength, or phase, multiple data pages can be superimposed. This feature is called $3 D$ storage. By illuminating the holographic medium with the reference beam from a proper incident angle, with a proper wavelength and phase, a certain object beam can be retrieved and then captured by the detector array at the receiving end. The detector array is usually a CCD or complimentary metal oxide semiconductor (CMOS) image sensor.

The mathematic channel model in [11] includes several key effects found in the actual HDS systems. In this model, multiple HDS channel impairments including IPI, fill factors of SLM and CCD pixels, limited contrast ratio, and noise are modeled. In this paper, we extend the channel model in [11] to include misalignment and oversampling. The block diagram of the modified channel model is depicted in Fig. 2.

The input binary data sequence, $A(i, j)$, takes on values in the set $\{1,1 / \varepsilon\}$, where $\varepsilon$ is a finite value called the amplitude contrast ratio. The SLM has a pixel shape function $p(x, y)$ given by

$$
p(x, y)=\Pi\left(\frac{x}{f f_{\mathrm{SLM}} \Delta}\right) \Pi\left(\frac{y}{f f_{\mathrm{SLM}} \Delta}\right)
$$

where $f f_{\text {SLM }}$ represents the SLM's linear fill factor, the symbol $\Delta$ represents the pixel pitch, and $\Pi()$ is the unit rectangular function. Another factor that contributes to IPI is the point spread function (PSF), a low-pass spatial behavior with impulse response $h_{A}(x, y)$ resulted from the limited aperture of the optics subsystem. This PSF is expressed as

$$
h_{A}(x, y)=h_{A}(x) h_{A}(y),
$$

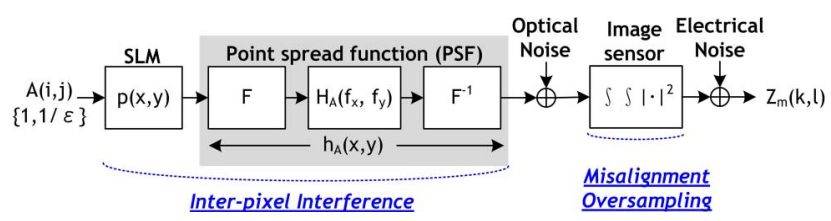

Fig. 2. (Color online) Block diagram of a complete channel model. 
where

$$
h_{A}(x)=\left(D / \lambda f_{L}\right) \operatorname{sinc}\left(x D / \lambda f_{L}\right) .
$$

Note that $D$ is the width of the square aperture, $\lambda$ is the wavelength of the incident light, and $f_{L}$ represents the focal length. The corresponding frequency-domain transfer function $H_{A}\left(f_{x}, f_{y}\right)$ in this case is the ideal $2 \mathrm{D}$ rectangular low-pass filter with a cut-off frequency equal to $D / 2 \lambda f_{L}$.

A CCD/CMOS image sensor is an inherently square-law integration device that detects the intensity of the incident light. The image sensor transforms the incoming signals from the continuous spatial domain to the discrete spatial domain. Quantization in space causes several errors due to offsets in sampling frequency, location, and orientation. Three misalignment effects, namely magnification, translation, and rotation, are modeled as $\left(\gamma_{x}, \gamma_{y}, \sigma_{x}, \sigma_{y}, \theta\right)$, where $\gamma_{x}, \gamma_{y}$ are the magnification factors in the $X$ and $Y$ directions, respectively; $\sigma_{x}, \sigma_{y}$ are the translation in the range of \pm 0.5 pixel along the $X$ and $Y$ directions, respectively; and the rotation angle, $\theta$, is positive in the counterclockwise direction. In addition, the oversampling ratio $M$, the pixel ratio between the SLM and CCD/CMOS sensor, is another factor to be considered. In the following study, we choose $M=2$ and adopt a misalignment condition with parameters of $\left(0.98,0.98,0.5,0.5,0.5^{\circ}\right)$, which is somewhat more severe than conventional settings, e.g., those used in $[\underline{7,12]}$.

Taking all the aforementioned effects into account, we have the final image sensor output at the $(k, l)$ th pixel position given by

$$
\begin{aligned}
C_{m}(k, l) & =\int_{\Lambda_{X} \Lambda_{Y}} \mid\left[\sum_{a} \sum_{b} A(i+a, j+b) h(x-a \Delta, y-b \Delta)\right] \\
& +\left.n_{o}\left(x^{\prime}, y^{\prime}\right)\right|^{2} \mathrm{~d} y^{\prime} \mathrm{d} x^{\prime}+n_{e}(k, l),
\end{aligned}
$$

where

$$
\begin{aligned}
& \Lambda_{X}=\left[\left(\frac{k-f f_{\mathrm{CCD}} / 2}{M \gamma_{x}}+\sigma_{x}\right) \Delta,\left(\frac{k+f f_{\mathrm{CCD}} / 2}{M \gamma_{x}}+\sigma_{x}\right) \Delta\right], \\
& \Lambda_{Y}=\left[\left(\frac{l-f f_{\mathrm{CCD}} / 2}{M \gamma_{y}}+\sigma_{y}\right) \Delta,\left(\frac{l+f f_{\mathrm{CCD}} / 2}{M \gamma_{y}}+\sigma_{y}\right) \Delta\right],
\end{aligned}
$$

$$
x^{\prime}=x \cos \theta+y \sin \theta, \quad y^{\prime}=-x \sin \theta+y \cos \theta .
$$

The subscript $m$ in $C_{m}(k, l)$ indicates misalignment; $h(x, y)$ in Eq. (4) is also known as pixel spread function (PxSF) and $h(x, y)=p(x, y) \otimes h_{A}(x, y) ; f f_{\mathrm{CCD}}$ represents the image sensor linear fill factor; $n_{o}\left(x^{\prime}, y^{\prime}\right)$ and $n_{e}(k, l)$ represent the optical noise and the electrical noise associated with the $(k, l)$ th pixel, respec- tively. Translation and magnification effects are represented by varying the range of integration square as in Eq. (5), while the rotation effect is represented by transforming $x-y$ axes to $x^{\prime}-y^{\prime}$ axes given by Eq. (6).

The optical noise, $n_{o}\left(x^{\prime}, y^{\prime}\right)$, results from optical scatter, laser speckle, etc., and is generally modeled as a circularly symmetric zero-mean Gaussian random process. On the other hand, the electrical noise, $n_{e}(k, l)$, arises from the electronics in the detector array and ensuing circuits, and is normally modeled as an additive white Gaussian noise with zero mean [13].

HDS systems dominated by electrical noise and those dominated by optical noise exhibit no major performance disparity in simulation results [11]. In light of this, we choose to consider the electricalnoise-dominated case in the following study. Signal-to-noise ratio $\left(\mathrm{SNR}_{e}\right)$ is used to characterize the extent of the electrical noise and is defined as

$$
\mathrm{SNR}_{e}=10 \log _{10}\left(0.5 / N_{0}\right),
$$

where $N_{0}$ is the variance of the electrical noise.

In the following investigation, we set some channel parameters as those specified in Table 1 according to the experimental HDS platform in [14]. The IPI effect is controlled by the normalized aperture width $w$ defined as $D \Delta / \lambda f_{L}$. Note that smaller aperture width causes higher degree of IPI. Two typical channel conditions are considered hereafter: $\mathrm{CH} 1$, with $w=1$, representing the case of medium IPI, and $\mathrm{CH} 2$, with $w=0.8$, representing the case of severe IPI. Throughout our discussion, channels taking misalignment and oversampling into consideration will be given clear indication of misalignment parameters and oversampling ratio.

\section{B. Pixel Detection}

In light of the aforementioned channel impairments, we designed a pixel detector that deals with all detrimental channel effects except noise. For misalignment compensation, we embed some training pixels in data pages to estimate misalignment parameters. With the estimated information of magnification, translation, and rotation as well as the predetermined oversampling ratio, a realignment and rateconversion filter can compensate for these effects and provide misalignment-free, pixel-matched images to the channel estimator and the modified 2D-MAP detector. The channel estimation block then

Table 1. Channel Parameters for Simulation

\begin{tabular}{cc}
\hline Parameters & Simulation Settings \\
\hline Page Size & $512 \times 512$ \\
$\lambda / f_{L} / \Delta$ & $515 \mathrm{~nm} / 89 \mathrm{~mm} / 18 \mu \mathrm{m}$ \\
$f f_{\mathrm{SLM}} / f f_{\mathrm{CCD}}$ & $0.9 / 0.9$ \\
$\varepsilon$ & 10 \\
$M$ & 2 \\
$\left(\gamma_{x}, \gamma_{y}, \sigma_{x}, \sigma_{y}, \theta\right)$ & $\left(0.98,0.98,0.5,0.5,0.5^{\circ}\right)$ \\
\hline
\end{tabular}


computes an estimated PxSF for the subsequent 2DMAP pixel detection. Finally, the modified 2D-MAP detector recovers the stored binary data. This work will focus on how the misalignment is compensated and assume that the degree of each misalignment is known. The signal flow and notations for variables used throughout the discussion are depicted in Fig. $\underline{3}$.

\section{Misalignment Compensation and Rate Conversion}

To remove the misalignment impairments and to convert the received signal rate to match the stored data pixel rate, we propose a low-complexity misalignment-compensation scheme that includes realignment as well as rate conversion. In the study, a metric called normalized mean squared error (NMSE) [15] is first defined for evaluating misalignment-compensation performance. The formula is given by

$$
\frac{\sum_{a, b \in E}(\tilde{Z}(a, b)-C(a, b))^{2}}{\sum_{a, b \in E} C(a, b)^{2}},
$$

where $C(a, b)$ denotes the $(a, b)$ th pixel value in the misalignment-free image; $E$ is the scope of the parameters $a$ and $b$ depending on the resolution of misalignment compensated images; and $\tilde{Z}(a, b)$ is the misalignment-compensation filter output:

$$
\begin{aligned}
& \tilde{Z}(a, b)= \\
& \left\{\begin{array}{ll}
Z_{m}(a, b), & \text { realignment }(\mathrm{Ra}) \\
Z(a, b), & \text { realignment }+ \text { rate conversion }(\mathrm{Ra}+\mathrm{Rc})
\end{array} .\right.
\end{aligned}
$$

Note that "Ra" and "Ra $+\mathrm{Rc}$ " indicate two stages in compensation: one with realignment only, the other one with both realignment and rate conversion.

\section{A. Realignment Interpolator}

Pixels in the captured images can be realigned by either 2D interpolators or 2D all-pass fractional delay filters [16]. For these realignment techniques, the realigned image quality can be improved by using filters with higher order, incurring higher computational complexity. In this paper, four practical realignment methods, namely bilinear interpolator, bicubic interpolator, truncated sinc interpolator, and truncated raised-cosine interpolator, are com-

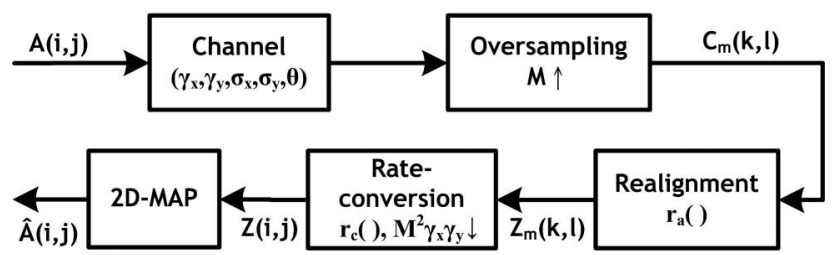

Fig. 3. Signal flows of pixels-detection blocks and related notations. pared in terms of both performance and computational complexity.

These four selected interpolators differ in filter order and tap coefficients. The bilinear interpolator performs $2 \mathrm{D}$ linear interpolation with the surrounding $2 \times 2$ pixels while the bicubic interpolator performs 2D 3rd-order interpolation taking into consideration the surrounding $4 \times 4$ pixels. On the other hand, a time-shifted sinc function with infinite interpolation length can achieve ideal interpolation. In practical scenarios, however, one can only implement a finite number of taps and thus the sinc function must be truncated. We consider also the raised-cosine interpolator with a truncated impulse response since this interpolator can have close-toconstant group delay. All the above 2D interpolators can be formulated as

$$
Z_{m}(k, l)=\sum_{(p, q) \in S} f\left(\mu_{x}-p\right) f\left(\mu_{y}-q\right) C_{m}\left(k^{\prime}+p, l^{\prime}+q\right),
$$

where $Z_{m}$ and $C_{m}$ are the realigned and misaligned images, respectively; $S$ is the input range of the $2 \mathrm{D}$ interpolators; $\mu_{x}$ and $\mu_{y}$ indicate the local fractional displacement from the nearest pixel $\left(k^{\prime}, l^{\prime}\right)$ in $C_{m}$, respectively; and $f()$ is the $1 \mathrm{D}$ impulse response of the corresponding interpolator. The relationship between the local fractional displacement $\mu_{x}$ and $\mu_{y}$ and the corresponding pixel position $k^{\prime}$ and $l^{\prime}$ on the misaligned image $C_{m}$ can be expressed as

$$
\begin{gathered}
\tilde{k}=\left(k \cos \theta-l \sin \theta+\sigma_{x}\right) \cdot M \gamma_{x}, \\
\tilde{l}=\left(k \sin \theta+l \cos \theta+\sigma_{y}\right) \cdot M \gamma_{y},
\end{gathered}
$$

$$
k^{\prime}=\lfloor\tilde{k}\rfloor, \quad \mu_{x}=\tilde{k}-k^{\prime}, \quad l^{\prime}=\lfloor\tilde{l}\rfloor, \quad \mu_{y}=\tilde{l}-l^{\prime} .
$$

The selected interpolators are evaluated based on two criteria, namely, all-pass frequency response and NMSE. An all-pass frequency response of a realignment interpolator is quite crucial for misalignment compensation since the HDS images often contain high spatial frequency components. Figure 4 shows $2 \mathrm{D}$ spatial frequency responses magnitude of the bilinear interpolator, the bicubic interpolator, the $2 \mathrm{D}$ truncated sinc interpolator, and the $2 \mathrm{D}$ truncated raised-cosine interpolator, all with typical $\left(\mu_{x}, \mu_{y}\right)$ setting. In addition, Fig. 5 shows the NMSE performance of the realignment interpolators under different degrees of misalignment.

With identical computational complexity, the bicubic interpolator and the $4 \times 4$-tap raised-cosine interpolator show a similar spatial frequency response magnitude, which presents better all-pass characteristic than that of the $4 \times 4$-tap sinc interpolator. In terms of the NMSE performance, the $4 \times 4$-tap 


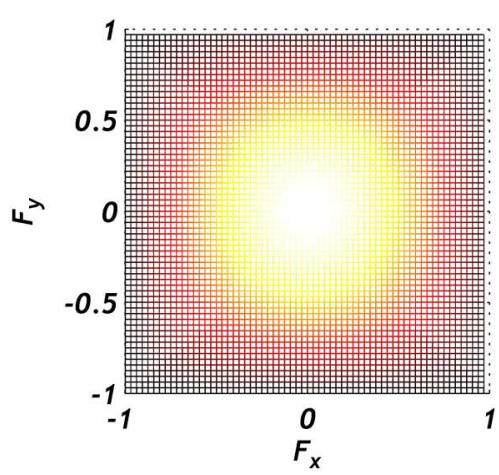

(a)

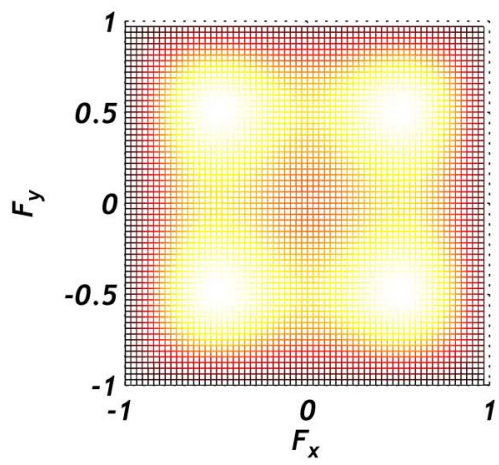

(c)
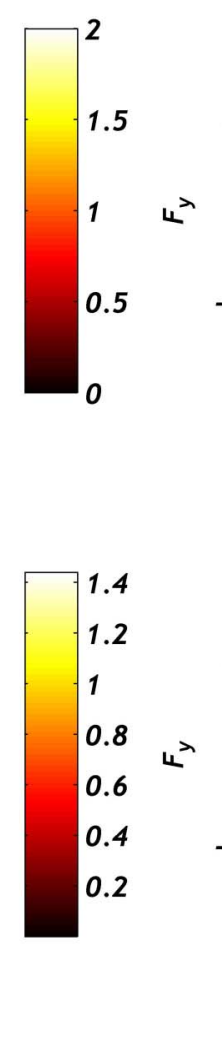

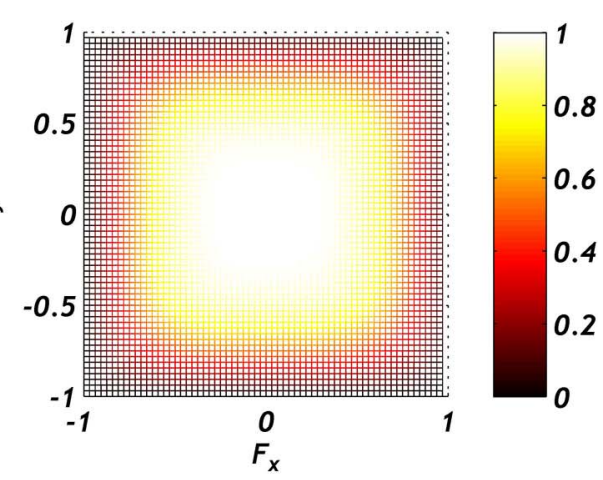

(b)

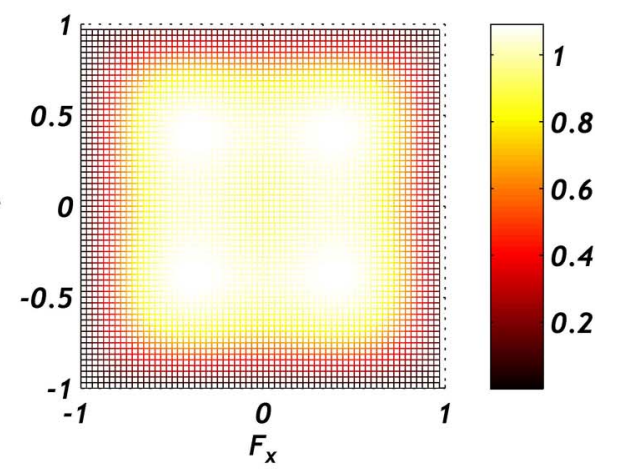

(d)

Fig. 4. (Color online) Two-dimensional frequency responses magnitude of (a) the bilinear interpolator, (b) the bicubic interpolator, (c) the $4 \times 4$-tap sinc interpolator, and (d) the $4 \times 4$-tap raised-cosine interpolator. $\left(\mu_{x}, \mu_{y}\right)=(0.5,0.5)$.

raised-cosine interpolator is better than the bicubic interpolator. By increasing the filter order, the truncated raised-cosine interpolator can further improve its NMSE performance, albeit with higher complexity. Considering the trade-off between performance and complexity shown in Fig. 6 , we adopt the $6 \times 6$-tap raised-cosine interpolator for realignment.

\section{B. Rate-Conversion}

Conventionally, misalignment compensation (e.g., [16]) considers only recovering displacement and rotation. The magnification effect and oversampling are oftentimes ignored. In conjunction with the realignment interpolator, we propose a rate-conversion filter to properly handle magnification and over-
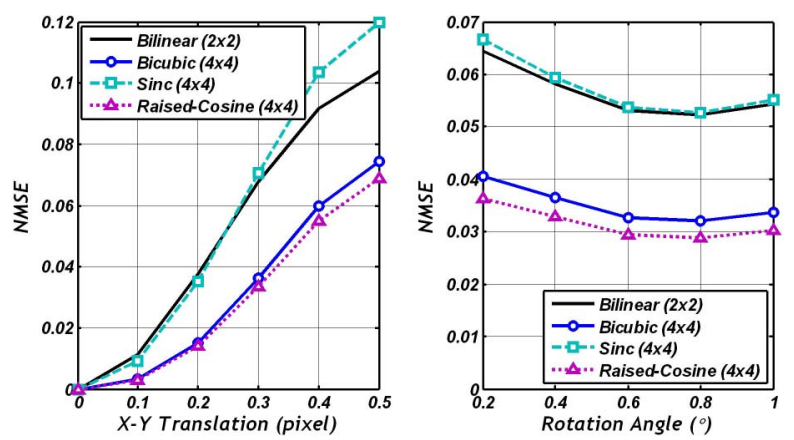

Fig. 5. (Color online) NMSE performance of four realignment interpolators under different translation and rotation effects using CH1. sampling and render pixel-matched images for ensuing pixel detection.

Based on the channel model in Eq. (4) we design a rate-conversion filter that linearly combines the surrounding pixels with proper weights. These weights are related to the respective area covered by the current pixel under consideration. Without loss of generality, the rate-conversion filter can be formulated

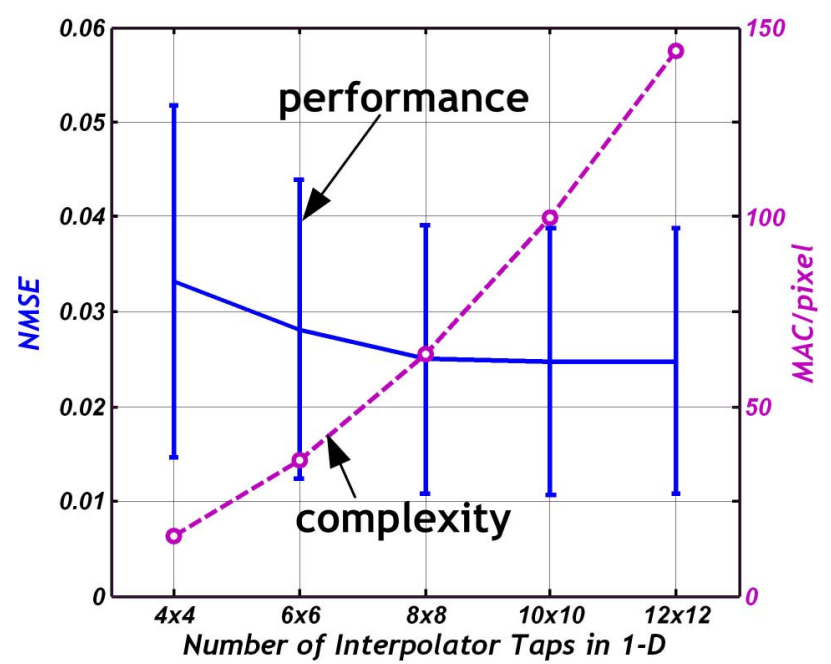

Fig. 6. (Color online) Trade-off between complexity and NMSE performance using the truncated raised-cosine interpolators with different sizes. The NMSE range is due to different setting of $\sigma_{x}, \sigma_{y}$, and $\theta$. 


$$
Z(i, j)=\sum_{p=P_{\min }}^{P_{\max }} \sum_{r=R_{\min }}^{R_{\max }} \nu_{x}(p) \nu_{y}(r) Z_{m}(k+p, l+r),
$$

where $Z_{m}$ indicates the realigned pixels, and the weights $\nu_{x}(p)$ and $\nu_{y}(r)$ depend on the magnification factors $\gamma_{x}$ and $\gamma_{y}$ as well as the oversampling ratio $M$. Usually with oversampling, $M \gamma_{x}$ or $M \gamma_{y}$ is larger than or equal to 1 , and $\nu_{x}(p)$ and $\nu_{y}(r)$ can be given by

$$
\nu_{x}(p)= \begin{cases}\left((k+p) / M \gamma_{x}\right) \bmod 1, & p=P_{\min } \\ \left(1 / M \gamma_{x}\right) \bmod 1, & P_{\min }<p<P_{\max } \\ 1-\sum_{a=P_{\min }}^{P_{\max }} \nu_{x}(a), & p=P_{\max }\end{cases}
$$

$$
\nu_{y}(r)= \begin{cases}\left((l+r) / M \gamma_{y}\right) \bmod 1, & r=R_{\min } \\ \left(1 / M \gamma_{y}\right) \bmod 1, & R_{\min }<r<R_{\max } \\ 1-\sum_{a=R_{\min }}^{R_{\max -1}} \nu_{y}(a), & r=R_{\max }\end{cases}
$$

respectively.

Note that the rectangular region with $\left(k+P_{\min }\right.$, $\left.l+R_{\min }\right)$ and $\left(k+P_{\max }, l+R_{\max }\right)$ as the lower-left and upper-right corners denotes those input pixels to be combined for computing the current output pixel at $(i, j)$ th position, where $(i, j)$ indicates the pixel index on the pixel-matched image $Z$, while $(k, l)$ is the pixel index on the realigned images $Z_{m}$. Furthermore, we set $P_{\max } P_{\min }+1=T_{x}$, where $T_{x}=\left\lceil M \gamma_{x}\right\rceil$ or $\left\lceil M \gamma_{x}\right\rceil+1$ and $R_{\max }-R_{\min }+1=T_{y}$, where $T_{y}=$ $\left|M \gamma_{y}\right|$ or $\left|M \gamma_{y}\right|+1$.

Significance of the rate-conversion filter is clear when we compare the simulated NMSE performance of the images $Z_{m}$ and $Z$. We use misalignment parameters of $\left(0.98,0.98,0.5,0.5,0.5^{\circ}\right)$ and an oversampling ratio of 2 . Clearly from Fig. $\underline{7}$, the rateconversion filter dramatically improves the NMSE
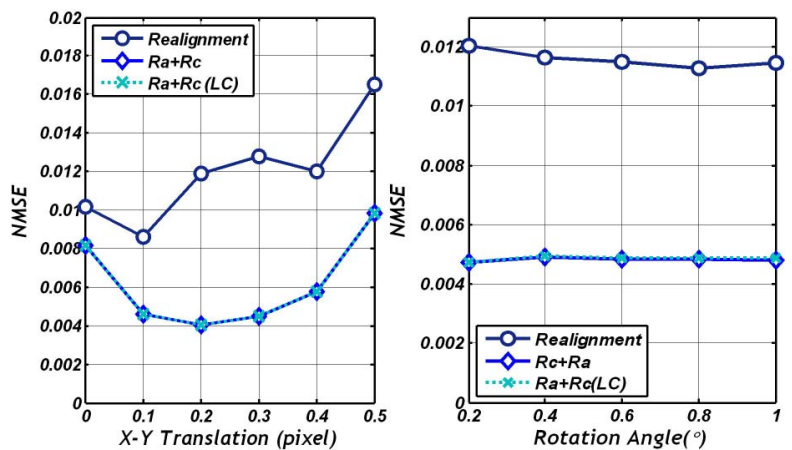

Fig. 7. (Color online) The NMSE performance of images that have undergone realignment only ( $\mathrm{Ra}$ ) and both realignment and rate conversion $(\mathrm{Ra}+\mathrm{Rc})$. "Ra $+\mathrm{Rc}(\mathrm{LC})$ " refers to the lowcomplexity implementation that applies two complexity-reduction schemes. performance, by up to $66 \%$. Consequently, rate conversion is indispensible in the processing of data images of the HDS systems.

\section{Low-Complexity Design}

For practical implementation, we propose two schemes to reduce the computational complexity of realignment and rate conversion. The first scheme partitions a $2 \mathrm{D}$ operation, such as realignment interpolation or rate conversion, into two $1 \mathrm{D}$ operations. As both the realignment interpolator and the rateconversion filter are separable (into product of two respective $1 \mathrm{D}$ operations), their implementation can be both achieved by two respective 1D operations [see Eqs. (10) and (13)]. The second scheme integrates the two $X / Y$ 1D realignment interpolators and the two $X / Y 1 \mathrm{D}$ rate-conversion filters to construct a misalignment-compensation block that consists of $1 \mathrm{D}$ compensation in the $X$ direction and 1D compensation in the $Y$ direction. The required kernel can be computed by convolving the two individual $1 D$ kernels.

The original 2D $K \times K$-tap realignment interpolator needs $K^{2}$ multiply-and-accumulate (MAC) operations for each pixel to be realigned. Breaking a 2D interpolator into two 1D interpolators each with $K$ taps reduces the number of MAC operations required to only $2 K$. Similarly, the $T_{x} \times T_{y}$-tap rate-conversion filter in Eq. (13) is also separable into two 1D filters. As a result, the computational complexity is reduced from $O\left(K^{2}\right)$ to $O(2 K)$ in realignment and from $O\left(T_{x} T_{y}\right)$ to $O\left(T_{x}+T_{y}\right)$ in rate conversion.

Another low-complexity scheme merges the $X$ direction $1 \mathrm{D}$ realignment interpolator with the $X$ direction 1D rate-conversion filter, and similarly for the $Y$ direction. The resultant filters have $\left(K+T_{x}-1\right)$ and $\left(K+T_{y}-1\right)$ taps in vertical and horizontal directions, respectively. The coefficients are calculated by convolving the two sets of coefficients. For example, to obtain the set of $\left(K+T_{x}-1\right)$ coefficients, $(K-1)\left(T_{x}-1\right)$ additions and $\left(K T_{x}\right)$ multiplications are needed for convolution.

The computational complexity required for the original $2 \mathrm{D}$ realignment plus $2 \mathrm{D}$ rate conversion and the misalignment-compensation blocks using the first reduction and both reduction schemes is summarized in Table 2 . As an example, the computation/memory requirements of a system with $K=6$ and $T_{x}=T_{y}=2$ using different implementations are listed in Table 3 . In this table, the complexity of the direct implementation (the $2 \mathrm{D}$ realignment interpolator followed by the $2 \mathrm{D}$ rate-conversion filter) is indicated as $100 \%$. About $84 \%$ reduction in addition and $74 \%$ reduction in multiplications can be achieved if both complexity-reduction schemes are applied.

Referring again to Fig. 7, the NMSE curve marked with "Ra + Rc (LC)" coincides with the "Ra + Rc" curve. This clearly indicates that the proposed lowcomplexity implementation of both realignment and rate conversion outperforms the traditional 
Table 2. Summary of the Computational Complexity Needed by Misalignment-Compensation Methods

\begin{tabular}{|c|c|c|c|}
\hline \multirow{2}{*}{ Compensation Method } & \multirow{2}{*}{ Implementation Scheme } & \multicolumn{2}{|c|}{ Computational Complexity } \\
\hline & & Additions/SLM Pixel & Multiplications/SLM Pixel \\
\hline $\mathrm{Ra}$ & - & $K^{2}$ & $K^{2}$ \\
\hline
\end{tabular}

misalignment mitigation that does not consider the magnification effect. Moreover, with techniques exploiting the separable property and convolution of kernels, the computational complexity of the proposed solution is lower than that of the traditional $2 \mathrm{D}$ realignment interpolator.

\section{2D-MAP Detection}

After removing the misalignment effects and generating pixel-matched images, the detector in the HDS system still needs to deal with IPI. In this section, we will propose several complexity-reduction schemes for a detection algorithm that has been shown to perform superbly for images under the influence of IPI.

\section{A. 2D-MAP Algorithm}

2D-MAP detection, proposed in [9] as the two-dimensional distributed data detection $\left(2 \mathrm{D}^{4}\right)$ algorithm, is actually the well-known max-Log-MAP algorithm. In this iterative algorithm, a log-likelihood ratio (LLR) for each pixel is always maintained during the iteration. More specifically, an LLR with a more positive value indicates a greater probability that $A(i, j)$, the data bit at the $(i, j)$ th pixel of the SLM image, is zero. On the contrary, a more negative LLR value indicates a greater probability that $A(i, j)=1$. Following the same reasoning, an LLR value around zero implies that $A(i, j)$ is equally likely to flip either way. As the iteration goes on, the LLR value at each pixel will be recalculated, based on the knowledge of previous LLRs of its eight neighbors. In all likelihood, this process makes each and every LLR move away from the origin and certainty about all pixels becomes higher and higher.

The procedure of the 2D-MAP detection comprises likelihood computation and update. The related likelihood computation formula is

$$
\begin{aligned}
\operatorname{LL}_{U}^{(k)}(i, j)= & \min _{N_{i j}}\left\{\frac{1}{2 N_{0}}\left|Z(i, j)-H\left(1, \mathbf{n}_{i j}\right)\right|^{2}\right. \\
& \left.+\mathbf{d}_{i j}^{(k-1)} \bullet \mathbf{n}_{i j}\right\}, \\
\operatorname{LL0}_{U}^{(k)}(i, j)= & \min _{N_{i j}}\left\{\frac{1}{2 N_{0}}\left|Z(i, j)-H\left(0, \mathbf{n}_{i j}\right)\right|^{2}\right. \\
& \left.+\mathbf{d}_{i j}^{(k-1)} \bullet \mathbf{n}_{i j}\right\} .
\end{aligned}
$$

Note that $H\left(A(i, j), \mathbf{n}_{i j}\right)$ is the IPI-inflicted channel output with $A(i, j)=0$ or 1 and a neighborhood pat- tern expressed as a binary vector, $\mathbf{n}_{i j}$, consisting of the eight binary pixels; $N_{i j}$ is the set of all possible neighborhood patterns. In addition, $\mathbf{d}^{(k-1)}$ is a vector consisting of the corresponding LLR values of neighboring pixels in the $(k-1)$ th iteration and the symbol "•" represents inner product of two vectors. In the above, all misalignment effects and oversampling have been properly handled and the only remaining channel effect is IPI. Updating of LLRs, the second step of the 2D-MAP, is executed at the end of each iteration. To avoid sudden changes in the updated LLR values, a forgetting factor $\beta$ is applied and the updated LLR takes the form of

$$
\begin{aligned}
L^{(k)}(i, j)= & (1-\beta) L^{(k-1)}(i, j) \\
& +\beta\left\{\operatorname{LL}_{U}^{(k)}(i, j)-\operatorname{LL}_{U}^{(k)}(i, j)\right\} .
\end{aligned}
$$

The value of $\beta$ can affect the speed and accuracy of the convergence. A larger $\beta$ leads to faster convergence and may result in poor detection performance. For more details, readers can refer to [9].

The 2D-MAP algorithm's consistent performance under moderate and severe IPI levels makes it a very good choice for pixel detection in the HDS systems. However, the problem of its high computational complexity still needs be resolved. Toward this end, we will present several effective complexity-reduction schemes for the 2D-MAP detection algorithm.

\section{B. Complexity Reduction}

2D-MAP detection offers superior performance under severe IPI and thus becomes a powerful solution to overcoming $2 \mathrm{D}$ crosstalk inherent in the HDS systems. However, the associated high computational complexity poses a hindrance in its practical realization. As a demonstration, all arithmetic operations involved in 2D-MAP detection based on a $3 \times 3$ neighborhood are unfolded and illustrated in Fig. 8. In each iteration, the normalized squared Euclidean distance and the sum of LLRs need be calculated for all 256 possible neighborhood patterns (candidates). In Fig. 8 , several effective schemes that

Table 3. Examples of Required Computational Complexity

\begin{tabular}{lrrrrr}
\hline$K=6, T_{x}=T_{y}=2$ & \multicolumn{2}{c}{ Addition } & & \multicolumn{2}{c}{ Multiplication } \\
\cline { 5 - 6 } \cline { 5 - 6 } Realignment only & 36 & - & & 36 & - \\
Ra + Rc (Direct) & 148 & $100 \%$ & & 148 & $100 \%$ \\
Ra + Rc (Scheme 1) & 52 & $35.14 \%$ & & 52 & $35.14 \%$ \\
Ra + Rc (Schemes 1 and 2) & 24 & $16.22 \%$ & & 38 & $25.68 \%$ \\
\hline
\end{tabular}




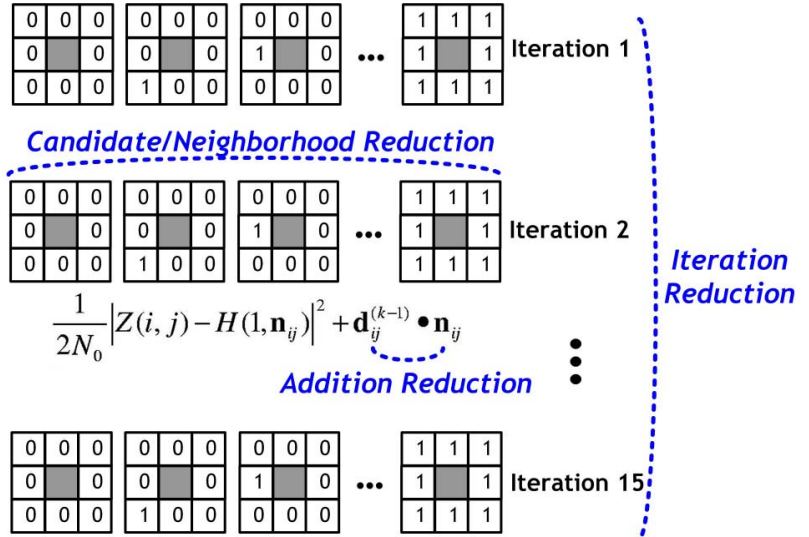

Fig. 8. (Color online) Arithmetic operations involved in 2D-MAP with $3 \times 3$ neighborhood and illustration of the four proposed schemes for complexity reduction.

reduce the complexity of the 2D-MAP detection algorithm are also depicted and will be described in the following.

\section{Iteration Reduction}

In an iterative algorithm such as 2D-MAP detection, the number of iterations needed to reach BER convergence depends on the channel condition. As the SNR gets higher/lower, fewer/more iterations are needed to attain the target BER. Hence, instead of executing a fixed number of iterations, the algorithm should be terminated as soon as further improvement in BER is not feasible. In this respect, we propose a novel criterion for early termination in the 2D-MAP detection algorithm.

A question naturally arises: how do we know when to stop the iteration? BER is not a suitable metric because it is often not available. Consequently, a metric that is easy to obtain and descriptive of the BER level must be devised. In the literature, there have been a few stopping criteria proposed to terminate iterative algorithms. The sign-change ratio (SCR) criterion and the hard-decision-aided (HDA) criterion are two such examples [17]. The SCR criterion counts the sign changes of extrinsic information and stops the iteration when the number of total sign-changes falls below a predefined threshold. The HDA criterion, on the other hand, terminates the iteration when the hard-decision results of a block between two consecutive iterations fully agree.

In the above stopping criteria, all pixels in a page or a region within a page terminate their iterative procedures simultaneously. In reality, some pixels converge faster, while others slower. Unlike convergence in BER, pixel convergence refers to the condition when the hard decision of that pixel stays at either " 0 " or " 1 " throughout future iterations. This absolute pixel convergence is difficult, if not impossible, to determine given only partial and local LLR information that the iterative 2D-MAP algorithm has. The question then becomes "Is there a simple and practical indicator that can more reliably decide if a pixel has converged?"

Given the formula of likelihood in Eq. (16), as the iterative procedure proceeds, the magnitudes of the LLR terms grow larger and larger. Therefore, belief about pixel polarities propagates and certainty about decisions gradually increases as the LLRs move away from the origin (i.e., the ambiguous state). Toward the final stage of the iterative procedure, the LLRs grow to a level that the sum of the neighborhood LLRs (the second term in Eq. (16)) dominates. When this happens, $\mathrm{LLO}_{U}$ and $\mathrm{LL} 1_{U}$ for this pixel will correspond to the same neighborhood pattern, $\mathbf{n}_{i j}$. In this case, this pixel has very likely already converged.

So we propose the following early termination criterion.

- For every pixel in the current iteration, record the two neighborhood patterns that result in the minimal $\mathrm{LLO}_{U}$ and minimal $\mathrm{LL}_{U}$. Denote these two neighborhood patterns as ML-C0 and ML-C1, respectively.

- If ML-C0 is equal to ML-C1 in two successive iterations, declare this pixel as "converged". Set its LLR to an extreme value, which, to other neighboring pixels, indicates that a decision about this pixel has been made. No further iterative detection for this pixel is needed.

- If a pixel does not reach the "converged" state before the predetermined number of iterations (15 in this case), stop its iterative procedure anyway.

The above early termination strategy can be illustrated in the flowchart shown in Fig. 9. A counter records the number of consecutive iterations in which the two ML candidates are the same.

\section{Candidate Reduction}

From Eq. (16), $2^{8}=256$ neighborhood patterns (candidates) must be searched to find the minimum likelihood in the 2D-MAP algorithm. After a close examination, we found that some candidates are unlikely to come out as the winner of the minimum operation. Consequently, these candidates need not be considered and computation associated with them is not necessary. In other words, only those probable candidates out of the 256 candidates need to enter the minimum operation and the outcome will not deviate much from that of a full search among all 256 candidates.

The candidate-reduction scheme is simple: only the candidates that are within a predetermined radius $\left(Q_{\max }\right)$ in Hamming distance to the current hard-decision neighborhood pattern will enter the minimum operation. This scheme is illustrated in Fig. 10(a), in which $Q$ represents the Hamming distance between a candidate and the current harddecision neighborhood pattern. The current harddecision neighborhood pattern is derived from the 


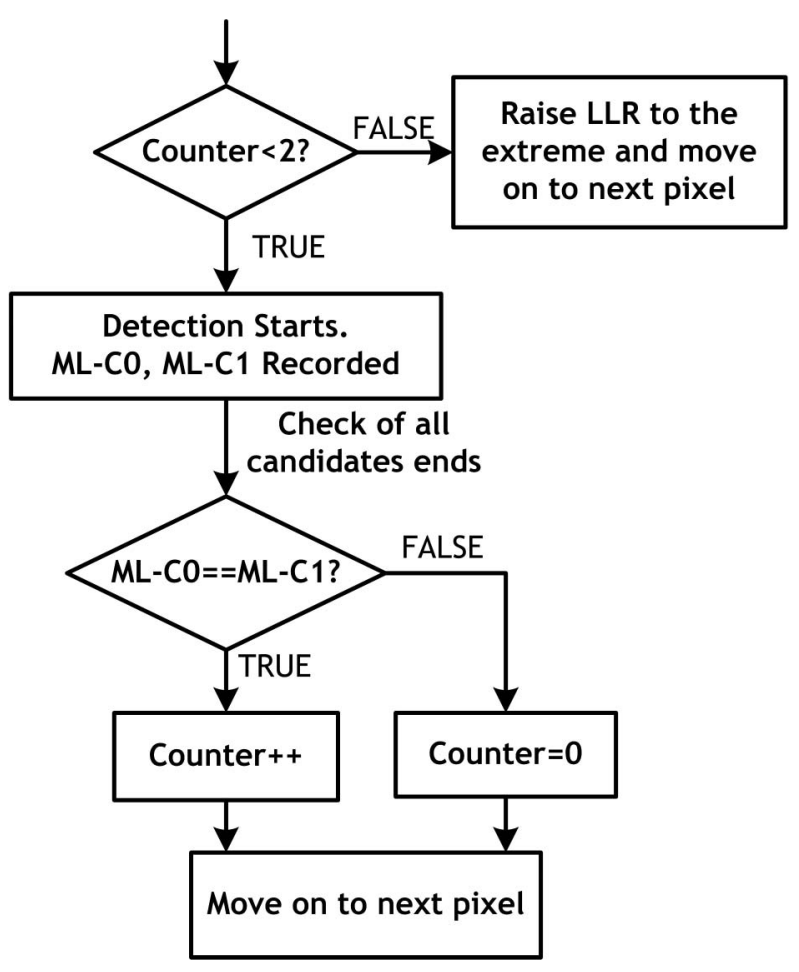

Fig. 9. Flowchart of the iteration-reduction scheme.

LLRs of those neighborhood pixels in the previous iteration.

To select a suitable radius $Q_{\max }$ in Hamming distance, the BER curves without error-correction coding have been plotted versus the radius, as shown in Fig. 10(b). This figure demonstrates a trade-off between the number of candidates and the uncoded BER performance. Taking both efficiency and performance into account, we set the radius to 2 . Therefore, the number of candidates to be processed is reduced from 256 to $C_{0}^{8}+C_{1}^{8}+C_{2}^{8}=37$, which is $85 \%$ reduction in computational complexity.

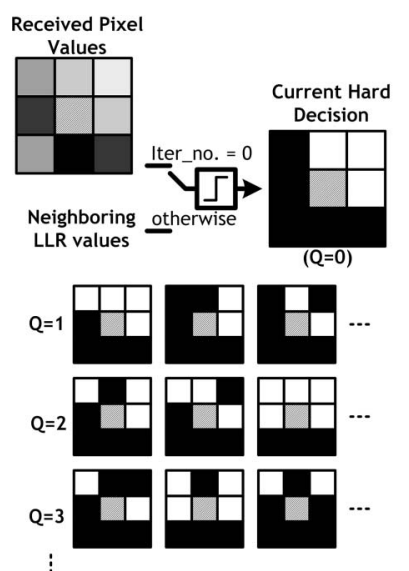

(a)

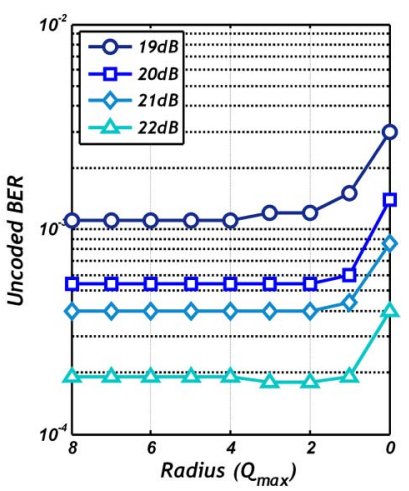

(b)
Fig. 10. (Color online) (a) Candidate-reduction scheme based on the Hamming distance; (b) trade-off between the number of candidates and uncoded BER performance for images with different SNR values.

\section{Neighborhood Reduction}

The neighborhood-reduction scheme combines the ideas of iteration reduction and candidate reduction. In addition to stopping the LLR calculation for itself, the early-terminated pixels are excluded from the LLR calculation of their neighbor pixels. In other words, the 2D-MAP's search range is shrunk as the early-terminated pixels are decided so they should be removed from the pattern during the search. As a result, the number of candidates is decreased. For example, if two pixels are converged and the $Q_{\max }$ is set as 2 , the number of test patterns is reduced from $C_{0}^{8}+C_{1}^{8}+C_{2}^{8}=37$ to $C_{0}^{6}+C_{1}^{6}+$ $C_{2}^{6}=22$. Moreover, we can adjust $Q_{\max }$ adaptively according to the number of decided pixels. Higher $Q_{\max }$ can be accepted when more pixels are early-terminated so that more candidates can be considered and the performance can be improved. Figure 11 illustrates examples of the neighborhood-reduction scheme when $Q_{\max }=2$ and $Q_{\max }=3$ with two converged pixels.

\section{Addition Reduction}

With candidate- and neighborhood- reduction schemes, the remaining candidates share many common terms in their sums of LLRs. We propose to first compute the LLR sum for the hard-decision neighborhood pattern, called $\mathrm{LX}_{\mathrm{hd}}$, and the sum of LLRs for other patterns with small $Q$ can be obtained by adding up to $Q_{\max }$ correction terms to $\mathrm{LX}_{\mathrm{hd}}$. More specifically, the addition-reduction scheme uses "tree-based LLR correction-term summation" described in the following.

Without loss of generality, we examine a case with $Q_{\max }=3$. First of all, the pixel positions of a neighborhood pattern are numbered as shown in Fig. 12 (a). Then the correction terms for all candidates within radius $\left(Q_{\max }=3\right)$ from the hard-decision neighborhood pattern can be computed more efficiently through a tree of additions, as shown in Fig. 12(b). In this tree, the first level corresponds to the candidates with $Q=1$, the second corresponds to those with $Q=2$, and so on. Traversing the tree downward

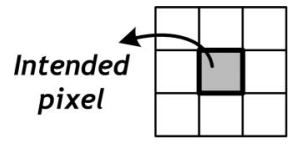

candidate \# = 37

(a)
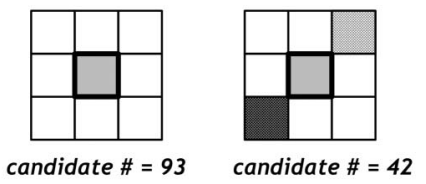

(b)
Fig. 11. Examples of neighborhood reduction. (a) $Q_{\max }=2$ and (b) $Q_{\max }=3$. 
one level corresponds to the addition of one more correction term. Without the hierarchical addition technique, it takes two additions to reach each and every node in the second level $(Q=2)$ and three additions for nodes in the third level $(Q=3)$. With partial sums of all intermediate-level nodes stored, many redundant additions are eliminated. In this way, on the average only one addition is needed for computing the sum of LLRs for a candidate, as summarized in Table 4.

Each of the aforementioned complexity-reduction schemes, except the neighborhood-reduction scheme, focuses on one computational aspect of the 2D-MAP algorithm and is independent of the others. In other words, each of them can be independently applied to the 2D-MAP algorithm. When combined, they result in significant reduction in the 2D-MAP algorithm complexity. In Fig. 13(a), the average iteration number of a 2D-MAP using the proposed early termination criterion is plotted. When compared with the original 2D-MAP algorithm with 15 iterations, the proposed iteration-reduction scheme reduces the total pixel iterations by up to one half. The iteration number is further reduced by nearly $2 / 3$ when the candidate-reduction and neighborhood-reduction schemes are applied. The average candidate number is also plotted in Fig. 13(a). The neighborhood-reduction scheme decreases $22 \%-30 \%$ of candidates by excluding the converged pixels in neighborhood patterns. Figure 13(b) depicts the uncoded BER performance of the modified 2D-MAP algorithm with the proposed complexity-reduction techniques. The performance degradation by applying the proposed schemes is negligible when compared to the conventional 2D-MAP algorithm with 15 iterations (marked as “iter $=15$ ").

Table 5 lists the improvement in computational complexity in terms of numbers of additions and multiplications by the above four schemes. In the simulation, the channel model with $w=0.8$ and $\mathrm{SNR}_{e}=18 \mathrm{~dB}$ is assumed. Assume that the computational complexity of the original 2D-MAP algo-
Table 4. Additions Needed to Generate Sum of LLRs for Candidates of Different $Q$

\begin{tabular}{lcccc}
\hline & $Q=0$ & $Q=1$ & $Q=2$ & $Q=3$ \\
\hline Number of candidates & 1 & 8 & 28 & 56 \\
Average addition per candidate & 3 & 1 & 1 & 1 \\
\hline
\end{tabular}

rithm with 256 candidates and 15 iterations is $100 \%$. As is clear from Table 5, the proposed complexity-reduction techniques can save up to $96.5 \%$ of additions and $95.2 \%$ of multiplications.

\section{System Simulation}

To consider the overall benefits of the techniques proposed in this work, we compare our proposed system with pixel detection using MMSE equalization, a commonly adopted scheme [18]. Basically, MMSE equalizers can eliminate misalignment and IPI simultaneously with adequate coefficients by inverting the channel response. However, MMSE equalizers, being a linear filter, always suffers from model mismatch as the inherent HDS channel is nonlinear.

Figure 14 plots the uncoded BER performance of several pixel detection methods for images corrupted by IPI only. The curves of adaptive threshold detection (marked with TH) under IPI-free channel and MMSE equalization (marked with MMSE) under IPI-inflicted channel are drawn for comparison with the 2D-MAP detection algorithm (2D). With all these complexity-reduction techniques, the modified 2D-MAP (marked with 2D*) still enjoys close-tooptimal detection performance. Note that the addition-reduction scheme does not introduce any performance degradation as it is mathematically equivalent to the original iteration formula of Eq. (16). BER degradation resulting from iteration, candidate-, and neighborhood-reduction schemes is negligible, as is evident from the simulated curves in Fig. 14.

Figure 15 illustrates the performance of the proposed pixel detection scheme under channel with

Temp. Hard Decision

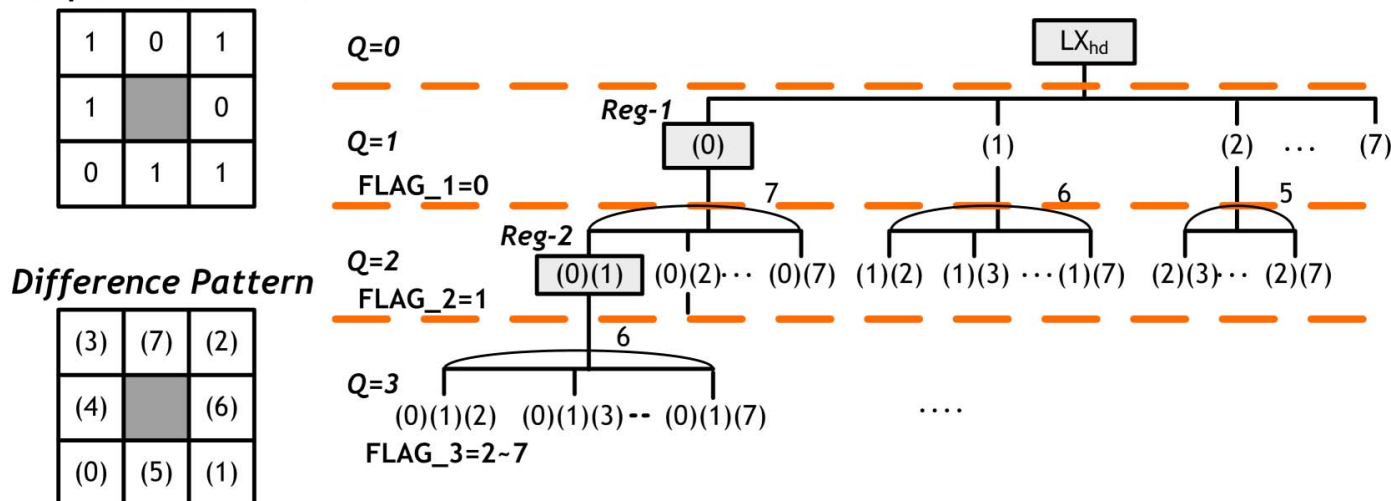

(a)

(b)

Fig. 12. (Color online) (a) Neighborhood pixel numbering. (b) Adder tree computes sum of LLRs more efficiently. 

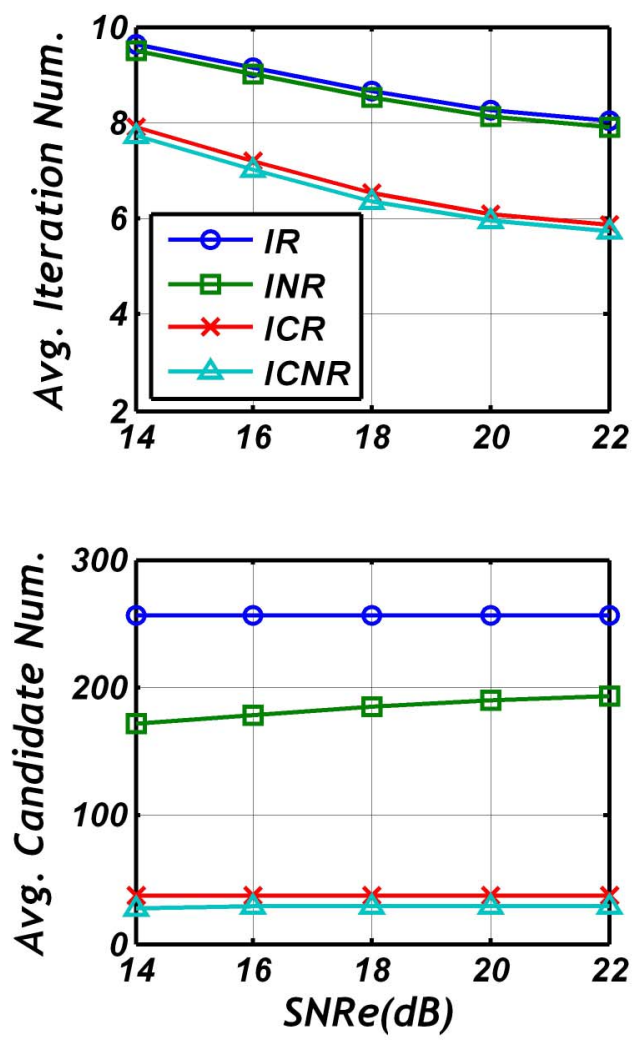

(a)

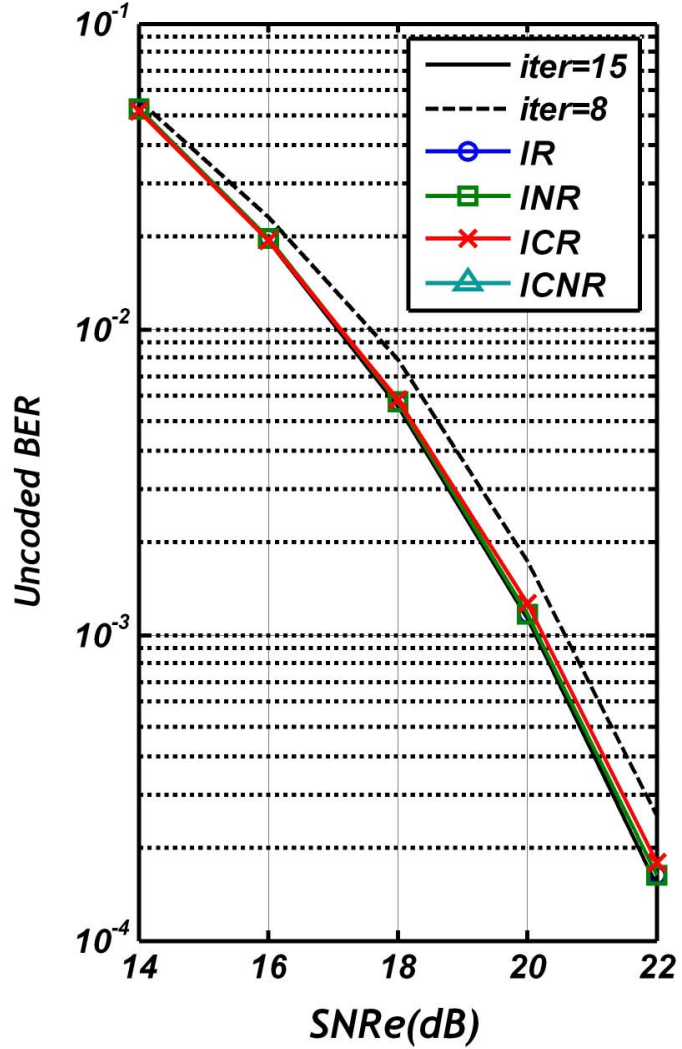

(b)

Fig. 13. (Color online) Performance after applying the neighborhood-reduction scheme: (a) average iteration number and average candidate number, (b) uncoded BER performance. $\mathrm{INR}=\mathrm{IR}+\mathrm{NR} . \mathrm{ICR}=\mathrm{IR}+\mathrm{CR} . \mathrm{ICNR}=\mathrm{IR}+\mathrm{CR}+\mathrm{NR} . Q_{\max }=2$ for $\mathrm{CR}$.

IPI as well as misalignment impairments (MA). The oversampling ratio is set to 2 to achieve acceptable BER performance. MMSE equalization can not achieve uncoded BER below $10^{-2}$ and thus is unable to deal with IPI and misalignment simultaneously. On the contrary, 2D-MAP-based methods (marked with $2 \mathrm{D}$ and $\left.2 \mathrm{D}^{*}\right)$ cope with the misalignmentplus-IPI channel (those marked with "MA") rather well and can have performance approaching that under the IPI-only channel, attesting to the efficiency and effectiveness of the proposed low-complexity pixel detection solution.

\section{Conclusions}

To detect data pixels embedded in images having undergone impairments in holographic data storage systems, e.g., IPI and misalignment, we proposed

Table 5. Summary of Computational Complexity-Reduction Schemes $^{a}$

\begin{tabular}{lcc}
\hline \multicolumn{1}{c}{$w=0.8 @ 18 \mathrm{~dB}$} & Addition & Multiplication \\
\hline Direct Implementation & $100 \%$ & $100 \%$ \\
$\mathrm{IR}$ & $57.7 \%$ & $57.7 \%$ \\
$\mathrm{IR}+\mathrm{CR}$ & $6.28 \%$ & $6.28 \%$ \\
$\mathrm{IR}+\mathrm{CR}+\mathrm{NR}$ & $4.82 \%$ & $4.82 \%$ \\
$\mathrm{IR}+\mathrm{CR}+\mathrm{NR}+\mathrm{AR}$ & $3.47 \%$ & $4.82 \%$ \\
\hline
\end{tabular}

${ }^{a} \mathrm{IR}=$ iteration reduction, $\mathrm{CR}=$ candidate reduction, $\mathrm{NR}=$ neighborhood reduction, and $\mathrm{AR}=$ addition reduction.

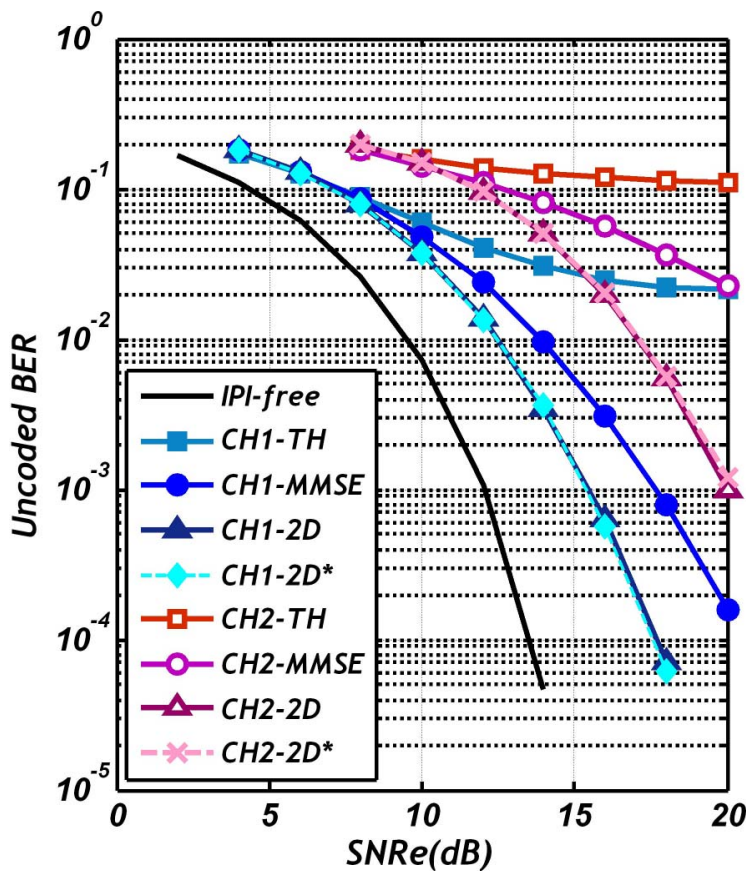

Fig. 14. (Color online) Uncoded BER performance of the 2D-MAP with four complexity-reduction schemes in two misalignment-free channels, $\mathrm{CH} 1$ and $\mathrm{CH} 2$. TH, adaptive threshold detection; 2D, 2D-MAP; 2D*, low-complexity 2D-MAP (IR + CR + NR + AR). 


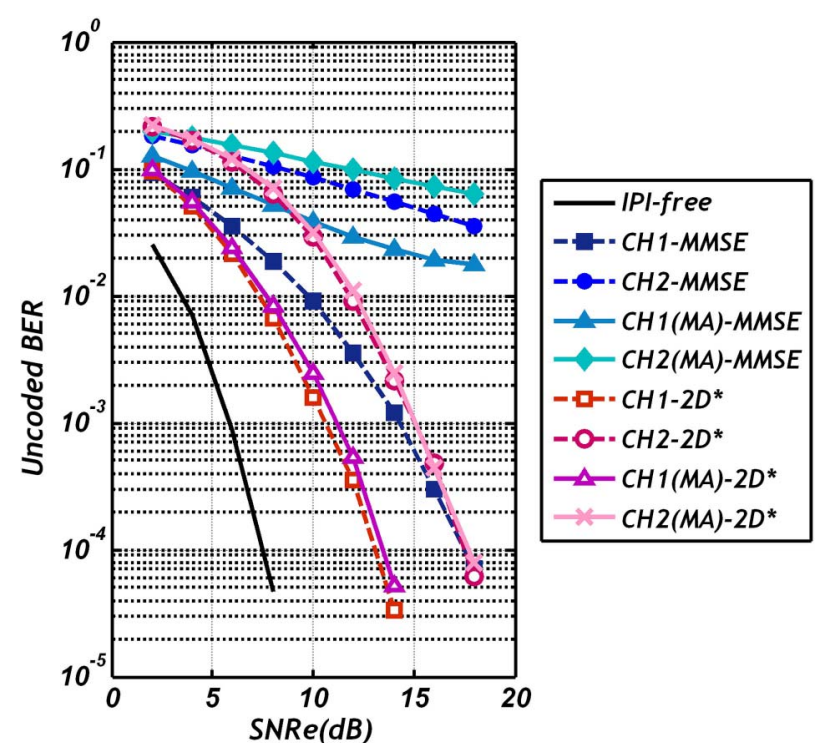

Fig. 15. (Color online) Performance of MMSE and 2D-MAP under IPI-only and misalignment-plus-IPI channel with oversampling ratio of 2 . The misalignment parameters for this simulation are $\left(0.98,0.98,0.5,0.5,0.5^{\circ}\right) .2 \mathrm{D}, 2 \mathrm{D}-\mathrm{MAP} ; 2 \mathrm{D}^{*}$, low-complexity $2 \mathrm{D}$ MAP; MA, proposed low-complexity misalignemnt compensation on images with misalignment effects.

in this paper a low-complexity solution that incorporates misalignment compensation and iterative $2 \mathrm{D}$ data detection. Misalignment compensation consists of realignment and rate conversion that remove three types of misalignment effects and produce pixel-matched images from oversampled sensor images. We further introduced efficient realization of this task by splitting a $2 \mathrm{D}$ operation to cascaded $1 \mathrm{D}$ operations and by merging realignment and rate conversion. In a typical example, $84 \%$ of the additions and $74 \%$ of the multiplications are saved without performance degradation.

Regarding IPI in the images of holographic data storage systems, 2D-MAP has been shown effective in combating it. This algorithm approximates the optimal ML detection by iteratively updating the LLR value on each pixel through combining information from the pixel itself and the associated neighborhood. This paper proposed several complexity-reduction schemes for the 2D-MAP detection implementation. Through adaptively determining the number of iterations and the search space, the iteration efficiency has been greatly improved. Common term extraction in summations further cuts down required arithmetic operations. In the end, the computational complexity of the modified low-complexity 2D-MAP algorithm is significantly decreased by up to $95 \%$ while the performance degradation is negligible when compared to the original 2D-MAP algorithm.
We are thankful for the support from Medratek, Inc., Taiwan and from the National Science Council of Taiwan (NSCT), under Grant no. NSC96-2220-E002-020.

\section{References}

1. H. J. Coufal, G. T. Sincerbox, and D. Psaltis, eds., Holographic Data Storage (Springer-Verlag, 2000).

2. L. Hesselink, S. S. Orlov, and M. C. Bashaw, "Holographic data storage systems," in Proceedings of the IEEE, Vol. 92, pp. 1231-1280 (2004).

3. H. Horimai and X. Tan, "Holographic information storage system: today and future," IEEE Trans. Magn. 43, 943-947 (2007).

4. L. Dhar, K. Curtis, and T. Fäcke, "Holographic data storage: coming of age," Nat. Photon. 2, 403-405 (2008).

5. L. Menetrier and G. W. Burr, "Density implications of shift compensation postprocessing in holographic storage systems," Appl. Opt. 42, 845-860 (2003).

6. S. G. Srinivasa and S. W. McLaughlin, "Signal recovery due to rotational pixel misalignment," in Proceedings of IEEE International Conference on Acoustics, Speech, and Signal Processing (2005), pp. IV-121-IV-124.

7. M. Ayres, A. Hoskins, and K. Curtis, "Image oversampling for page-oriented optical data storage," Appl. Opt. 45, 24592464 (2006).

8. M. Keskinoz and B. Kumar, "Application of linear minimum mean-squared-error equalization for volume holographic data storage," Appl. Opt. 38, 4387-4393 (1999).

9. X. Chen, K. M. Chugg, and M. A. Neifeld, "Near-optimal parallel distributed data detection for page-oriented optical memories," IEEE J. Sel. Top. Quantum Electron. 4, 866-879 (1998).

10. B. M. King and M. A. Neifeld, "Parallel detection algorithm for page-oriented optical memories," Appl. Opt. 37, 6275-6298 (1998).

11. M. Keskinoz and B. Kumar, "Discrete magnitude-squared channel modeling, equalization, and detection for volume holographic storage channels," Appl. Opt. 43, 1368-1378 (2004).

12. W. Chou and M. A. Neifeld, "Interleaving and error correction in volume holographic memory systems," Appl. Opt. 37, 69516968 (1998).

13. C. Gu, F. Dai, and J. Hong, "Statistics of both optical and electrical noise in digital volume holographic data storage," IEEE Electron. Lett. 32, 1400-1402 (1996).

14. G. W. Burr, J. Ashley, H. Coufal, R. K. Grygier, J. A. Hoffnagle, C. M. Jefferson, and B. Marcus, "Modulation coding for pixelmatched holographic data storage," Opt. Lett. 22, 639641 (1997).

15. M. Goldberg and H. Sun, "Image sequence coding using vector quantization," IEEE Trans. Commun. 34, 703-710 (1986).

16. K. J. Pharris, "Methods and systems for holographic data recovery," U.S. patent application 20050018263 (27 January, 2005).

17. R. Y. Shao, S. Lin, and M. P. C. Fossorier, "Two simple stopping criteria for turbo decoding," IEEE Trans. Commun. 47, 11171120 (1999).

18. M. R. Ayres, A. Hoskins, and K. R. Curtis, "Processing data pixels in a holographic data storage system," WIPO patent WO/2006/093945 (8 September, 2006). 